\title{
Positive and negative emotional arousal increases duration of memory traces: common and independent mechanisms
}

\author{
F. Cruciani ${ }^{1}$, A. Berardi ${ }^{1}$, S. Cabib ${ }^{1,2}$ and D. Conversi ${ }^{1,2 *}$ \\ ' Department of Psychology, Center D. Bovet, University "Sapienza," Rome, Italy \\ ${ }^{2}$ Fondazione Santa Lucia Istituto di Ricovero e Cura a Carattere Scientifico, European Centre for Brain Research, Rome, Italy
}

\section{Edited by:}

Antonella Gasbarri, University of

L'Aquila, Italy

\section{Reviewed by:}

James L. McGaugh, University of California at Irvine, USA

Pedro Bekinschtein, University of Cambridge, UK

*Correspondence:

D. Conversi, Department of Psychology, Center D. Bovet, University "Sapienza," via dei Marsi 78, Rome 1-00185, Italy. e-mail: david.conversi@uniroma1.it
We compared the ability of positive and negative emotional arousal to increase the duration of consolidated memory traces. Positive arousal was modulated by manipulating the motivational salience of the testing cage of an object recognition test. Negative emotional arousal was modulated by manipulating shock levels in a step-through inhibitory avoidance (IA). Mice trained in either a high (chocolate-associated) or a low (inedible object-associated) emotionally arousing cage showed discrimination of a novel object $24 \mathrm{~h}$ after training, but only mice trained in the more arousing cage showed retention $96 \mathrm{~h}$ after training. Mice trained with either low $(0.35 \mathrm{~mA})$ or high $(0.7 \mathrm{~mA})$ shock intensities showed increased stepthrough latencies when tested $24 \mathrm{~h}$ after training, but only mice trained with the higher shock intensity showed retention of the IA learning 1 week after training. Administration of the phosphodiesterase type IV inhibitor Rolipram immediately after training in the two low arousing conditions increases duration of both responses.

Keywords: long-term memory, persistence, emotional arousal, hedonic valence, object recognition, inhibitory avoidance, cAMP, Rolipram

\section{INTRODUCTION}

The establishment of long-term memory (LTM) requires the stabilization of learning-induced synaptic changes. This process is referred to as memory consolidation (McGaugh, 1966; Dudai, 1996, 2002, 2004; Lechner etal., 1999; Dudai and Eisenberg, 2004). However, not all long-term "consolidated" memories last equally. Indeed, everyday experience shows that duration of LTM is highly variable. The behavioral and brain factors underlying this variability are poorly understood.

A large body of evidence indicates that emotional arousal enhances LTM duration by acting on memory consolidation (for review see McGaugh, 2006). However, whether this effect is independent of the hedonic valence (positive or negative) of arousing experience is unknown. Indeed, testing this hypothesis is hampered by the lack of one-trial tasks specifically designed to model the effects of positive arousal on LTM duration. Thus, the first aim of the present study was to develop a behavioral protocol able to induce differentially persistent LTM traces in mice, depending on the magnitude of positive emotional arousal induced by the testing cage during a single learning trial. To this aim, the novel object recognition test (ORT) was used as a one-trial, non-aversive, learning task (Ennaceur and Delacour, 1988; Ennaceur, 2010) and the emotional salience of the testing cage was modulated by repeatedly pairing it with a highly palatable food (chocolate).

Recently, it has been observed that intra-hippocampal infusion of the 8-bromoadenosine- $3^{\prime}, 5^{\prime}$-cyclic monophosphate (8BrcAMP) analog of cyclic adenosine monophosphate (cAMP) immediately after a weak (low arousing) inhibitory avoidance (IA) training mimics the effect of a strong (high arousing) training by converting a rapidly decaying LTM into a long-lasting one (Rossato et al., 2009). This finding suggests that the enhancement of LTM duration induced by a high emotional arousal may be mediated by cAMP-dependent signaling around the time of memory formation. However, it is unknown whether cAMP-dependent signaling is involved in arousal-induced enhancement of LTM duration regardless of the hedonic valence of the learning experience. Thus, the second aim of the present study was to comparatively evaluate the role of cAMP-dependent signaling in the modulation of LTM duration in both the positive task here reported and in a negative standard one (IA). To this aim, the phosphodiesterase type IV inhibitor Rolipram, known to enhance intracellular cAMP concentration, was administered in a single peripheral injection after low arousing training conditions that induce, in both tasks, only non-persistent LTM traces (i.e., lasting $24 \mathrm{~h}$ but not beyond $96 \mathrm{~h}$ ).

\section{MATERIALS AND METHODS SUBJECTS}

Male CD1 mice (Harlan Laboratories, Udine, Italy) were purchased at 6 weeks of age and housed four to a cage on a 12-h light-dark cycle (lights on at 7:00 a.m.). Food and water were available ad libitum except when food deprivation was required by design (see Materials and Methods). Mice were left undisturbed for 2 weeks before behavioral testing. Experiments were carried out in accordance with the Italian national law (DL 116/92) on the use of animals in research.

\section{DRUGS}

Rolipram (Sigma-Aldrich, Milano, Italy) was freshly suspended in $5 \%$ ethanol and $95 \%$ saline solution on every experimental day (Rutten et al., 2006). Rolipram was injected intraperitoneally 
(i.p.) in a volume of $10 \mathrm{ml} / \mathrm{kg}$ at the following doses: 0,3 , and $10 \mathrm{mg} / \mathrm{kg}$. These doses were chosen because they have been shown to be rapidly effective to elevate cAMP concentration in the mouse brain (Randt etal., 1982). For either IA or object recognition experiments, Rolipram was administered immediately or $120 \mathrm{~min}$ post-training.

\section{BEHAVIORAL PROCEDURES Object recognition}

Mice underwent ORT in a custom made apparatus consisting of a square box $(60 \mathrm{~cm} \times 60 \mathrm{~cm} \times 30 \mathrm{~cm})$ made of black Plexiglas ${ }^{\circledR}$ subdivided in four equal arenas. The floor of the arenas was made of textured transparent Plexiglas ${ }^{\circledR}$ surmounting a plate of opalescent Plexiglas $^{\circledR}$ thick $1 \mathrm{~cm}$. The apparatus was dimly illuminated by halogen light sources placed below it (Nilsson et al., 2007). Light sources (four) were carefully positioned approximately $50 \mathrm{~cm}$ under each corner of the apparatus and directed toward the room floor. This allowed an homogeneous illumination throughout all arenas ( 10 lux, measured with a PCE-EM882 multimeter from http://www.pce-italia.it). The apparatus was placed in a ventilated sound-attenuated cabinet. Objects to discriminate were four types, one type for arena: white wooden cube, gray plastic cylinder, gray plastic sphere, and silver metallic parallelepiped. They were available in triplicate copy and, based on pilot studies, all aroused comparable levels of exploration in CD1 mice (data not shown). For training phase, objects were placed at two opposite corners of the arenas $(6.5 \mathrm{~cm}$ from the object center to the corner). To prevent mice from displacing objects during testing, they were temporarily fixed to the floor of the arenas with repositionable adhesive pastels (UHU patafix white). On the training trial, mice were allowed to freely explore two identical objects (sample objects: A1 and A2) for $15 \mathrm{~min}$. On the test trial, the third copy of the familiar object (A3) and a novel object (B) were placed in the same location of training and mice were allowed to freely explore them for $10 \mathrm{~min}$. Cohorts of four mice belonging to the same cage and to the same experimental group were tested simultaneously. Each mouse was tested only once. All combinations and locations of objects were counterbalanced to reduce potential biases because of preference for particular locations or objects. Between each session, apparatus and objects were thoroughly cleaned with $70 \%$ ethanol to remove urine and fecal boli and to homogenize olfactory trails. Mice behavior was recorded with a camera connected to a Debian GNU/Linux ${ }^{1}$ workstation equipped with hardware MPEG encoding capabilities. MPEG videos were blindly analyzed by experienced observers. Object exploration was defined as pointing the nose to the object at a distance of $1 \mathrm{~cm}$ and/or touching it with the nose. Turning around, climbing, or sitting on an object was not considered as exploration.

\section{Experiment 1}

This experiment was aimed to set-up a model to evaluate the effect of positive emotional arousal on LTM duration in mice and to evaluate the relative role of acute food deprivation and of the caloric and/or fat properties of chocolate in that model.

${ }^{1}$ http://www.debian.org
Effect of positive arousal on long-term memory duration. Sixtyfour mice were pre-exposed for $15 \mathrm{~h}$ to milk chocolate (Lindt, two pieces for cage, each weighing approximately $2 \mathrm{~g}$ ) mixed to standard food in the home-cage from 7:00 p.m. of the day before the starting of behavioral testing. Then, mice underwent a delay conditioning phase lasting 4 days. This phase comprised two daily sessions on days 1-3 (10:00 a.m. and 2:00 p.m.) and a single session on day 4 (10:00 a.m.). Sessions were as follows: mice were allowed to freely explore the empty arenas for $10 \mathrm{~min}$ then, depending on the experimental group, chocolate pieces (CHOC, $n=16$ ) or black Lego ${ }^{\circledR}$ blocks (OBJ, $n=16$ ), approximately of the same form and size of chocolate pieces, were dropped in the arenas by the experimenter (one stimulus for arena). Mice were allowed to interact with these stimuli for additional $5 \mathrm{~min}$ (total session duration: $15 \mathrm{~min}$ ). At 7:00 p.m. of day 3 all mice were fooddeprived. On day 4 at 2:00 p.m. all mice underwent ORT training. Training session differed from previous ones only for the presence of sample objects in the arenas. Immediately after training, mice were returned to their home cages and they were given ad libitum food. Half mice from each group were tested for sample object recognition $24 \mathrm{~h}$ after training, the remainder were tested $96 \mathrm{~h}$ after training. At 7:00 p.m. of the day before test session all mice were food-deprived.

Control of the role of acute food deprivation. Forty-eight mice were submitted to the same protocol as the CHOC group except for the following differences: a first group $(n=16$, FF-FF) was never food-deprived; a second group ( $n=16$, FD-FF) was fooddeprived $15 \mathrm{~h}$ before training but not before test; a third group ( $n=16, \mathrm{FF}-\mathrm{FD})$ was food-deprived $15 \mathrm{~h}$ before test but not before training. CHOC group was renamed FD-FD when compared to FF-FF, FF-FD, and FD-FF groups. Mice from these groups were tested for sample object recognition $96 \mathrm{~h}$ after training.

Control of the role of schedule of chocolate exposure. Thirty-two mice were treated identically to the CHOC group but, half (PRE) received chocolate only during conditioning phase, the remainder (POST) received chocolate only during the last $5 \mathrm{~min}$ of training. CHOC group was renamed PRE + POST when compared to PRE and POST groups. Mice from these groups were tested for sample object recognition $96 \mathrm{~h}$ after training.

\section{Experiment 2}

This experiment was aimed to evaluate the ability of Rolipram to enhance LTM duration in the OBJ condition. Thirty-two mice were randomly assigned to this experiment. Mice were treated identically to the OBJ group of Experiment 1. Three groups of mice $(n=8)$ were administered, respectively, vehicle, 3 or $10 \mathrm{mg} / \mathrm{kg}$ immediately after training. One group $(n=8)$ were administered $3 \mathrm{mg} / \mathrm{kg}$ of Rolipram $120 \mathrm{~min}$ after training. Mice were tested for sample object recognition $96 \mathrm{~h}$ after training. Lowest effective Rolipram dose has been previously determined in pilot studies (data not shown).

\section{INHIBITORY AVOIDANCE}

Mice were trained and tested in a step-through IA task. The apparatus consisted of a trough-shaped alley $(91 \mathrm{~cm}$ long, $15 \mathrm{~cm}$ deep) divided into two compartments by a retractable door: an 
illuminated safe compartment $(31 \mathrm{~cm}$ long) and a dark shock compartment ( $60 \mathrm{~cm}$ long). Mice were not habituated to the dark compartment before the training trial. On the training trial, each mouse was placed in the lit start compartment facing away from the shock compartment. After the mouse stepped with all four paws into the dark compartment, the retractable door was closed and an inescapable foot-shock $(0,0.35$, or $0.7 \mathrm{~mA}, 50 \mathrm{~Hz}, 2.0 \mathrm{~s})$ was administered. The mouse was removed from the dark compartment $30 \mathrm{~s}$ after termination of the foot-shock. The lowest shock intensity was chosen on the basis of previous studies showing that it is sufficient to induce IA retention for at least $24 \mathrm{~h}$ in mice (Baarendse et al., 2008). The highest shock intensity was chosen on the basis of a previous study in mice showing that it induces IA retention beyond $24 \mathrm{~h}$ (Boccia et al., 2004). On the retention trial, each mouse was placed into the light compartment with the retractable door open and allowed to explore the box freely. The latency to enter the dark compartment with all four paws was recorded with a timer by a nearby experimenter as a measure of retention. Retention test ended either if the mouse stepped into the dark compartment or if it failed to cross within $180 \mathrm{~s}$. In the latter case, mouse was assigned a score of $180 \mathrm{~s}$. Foot-shock was omitted on retention test. Each mouse was tested only once.

\section{Experiment 3}

This experiment was aimed to test in mice the effect of magnitude of negative arousal on LTM duration (Bekinschtein et al., 2007). Ninety-six mice were randomly assigned to this experiment. Onethird of mice were pseudo-trained by omitting the shock, onethird were trained with the $0.35 \mathrm{~mA}$ shock, and the remainder were trained with the $0.7 \mathrm{~mA}$ shock. Half of the mice for each shock condition $(n=16)$ were tested for retention $24 \mathrm{~h}$ after training, the other half were tested 1 week after training.

\section{Experiment 4}

This experiment was aimed to evaluate the ability of Rolipram to enhance LTM duration in the low shock condition (0.35 mA). Sixty mice were randomly assigned to this experiment. Four groups of mice $(n=12)$ were trained with the $0.35 \mathrm{~mA}$ shock, then they were administered vehicle, 3 or $10 \mathrm{mg} / \mathrm{kg}$ of Rolipram immediately post-training or they were administered $10 \mathrm{mg} / \mathrm{kg}$ of Rolipram 120 min post-training. In order to rule out a nonspecific drug effect a fifth group $(n=12)$ was pseudo-trained and it was administered $10 \mathrm{mg} / \mathrm{kg}$ of Rolipram immediately after being removed from the dark compartment. All mice were tested for retention 1 week post-training. Lowest effective Rolipram dose has been previously determined in pilot studies (data not shown).

\section{DATA ANALYSIS}

Statistical analyses were performed on a Debian GNU/Linux workstation using the $\mathrm{R}$ free software environment for statistical computing $^{2}$ and its graphical interface R-Commander (Fox, 2005). An error probability level of $p<0.05$ was accepted as statistically significant.

${ }^{2}$ http://www.r-project.org/

\section{Object recognition}

Data from Experiment 1 were analyzed using: (1) two-way analysis of variance (ANOVA) for independent factors to compare $\mathrm{CHOC}$ and $\mathrm{OBJ}$ mice either at 24 or $96 \mathrm{~h}$ post-training; (2) two-way ANOVA for independent factors to compare CHOC mice free-fed (FF) or food-deprivated (FD) either at training or at test; (3) oneway ANOVA to compare mice that, except for initial pre-exposure, received chocolate only during conditioning phase (PRE), only after training (POST), both during conditioning and after training (PRE + POST). In case of significant two-way interaction simple effect analysis was performed with the Student's $t$-test for independent samples (Welch variant, two-tailed). In case of significant overall one-way $F$ ratio, ANOVA was followed by Tukey's post hoc tests.

Data from Experiment 2 were analyzed using: (1) one-way ANOVA to compare mice treated with different Rolipram doses $(0,3$, and $10 \mathrm{mg} / \mathrm{kg}) ;(2)$ Student's $t$-test for independent samples (Welch variant, two-tailed) to compare vehicle-treated mice and mice treated with $3 \mathrm{mg} / \mathrm{kg}$ Rolipram $120 \mathrm{~min}$ post-training). For all experiments, one sample $t$-tests (two-tailed) were used to determine whether the discrimination index (D.I.) of each experimental group was different from 0 (chance level). Mice showing less than $5 \mathrm{~s}$ of total object exploration during ORT test were excluded from analysis (Sik et al., 2003).

\section{Inhibitory avoidance}

Because a cut-off of $180 \mathrm{~s}$ was imposed during test sessions, the step-through latency was expressed as median and inter-quartile range and analyzed with non-parametric tests. Kruskal-Wallis ANOVA was used when comparing more than two groups. Two-sample Wilcoxon test (two-tailed) was used either when comparing two groups or as post hoc test following a significant overall Kruskal-Wallis test.

\section{RESULTS}

\section{OBJECT RECOGNITION}

\section{Experiment 1}

Effect of magnitude of positive arousal on long-term memory duration. Two-way ANOVA for total exploration time did not reveal significant effects nor interaction for factors "stimulus paired with test context" and "inter-trial interval". Figure 1A shows the D.I. measured 24 and $96 \mathrm{~h}$ after training as a function of stimulus paired with test context. Two-way ANOVA for D.I. revealed a significant interaction between stimulus paired with test context and inter-trial interval $\left(F_{1,60}=5.06, p<0.05\right)$. For $24 \mathrm{~h}$ test, simple effect analysis did not reveal a significant D.I. difference between $\mathrm{CHOC}$ and OBJ groups and one sample $t$-tests revealed that D.I. from both groups was significantly above chance level (CHOC: $t_{15}=3.56, p<0.005$; OBJ: $t_{15}=5.46, p<0.0001$ ). For $96 \mathrm{~h}$ test, simple effect analysis revealed that OBJ group had a lower D.I. respect to CHOC group $\left(t_{30}=3.02, p<0.01\right)$. Moreover, simple effect analysis for each level of stimulus paired with test context revealed that only the D.I. of OBJ group at $96 \mathrm{~h}$ test was lower than that of $24 \mathrm{~h}$ test $\left(t_{30}=2.42, p<0.05\right)$. One sample $t$-tests revealed that only D.I. from CHOC group was significantly above chance level $\left(t_{15}=7.24, p<0.0001\right)$. These results indicate that long-term duration of object memory is enhanced when 


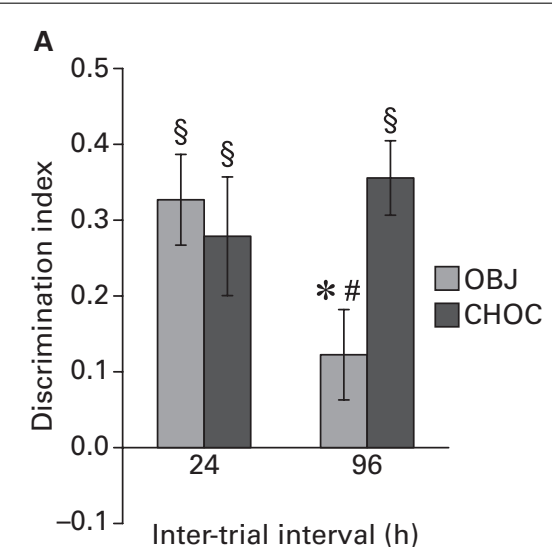

FIGURE 1 | (A) Discrimination index (mean \pm SEM) as a function of stimulus paired with test context measured 24 or $96 \mathrm{~h}$ post-training $\left({ }^{*} \mathrm{vs} \mathrm{CHOC}\right.$, $p<0.01$; \#vs 24 h, $p<0.05$; ${ }^{\S}$ vs $0, p<0.0001$ ); (B) Discrimination index (mean \pm SEM) as a function of feeding state before behavioral testing

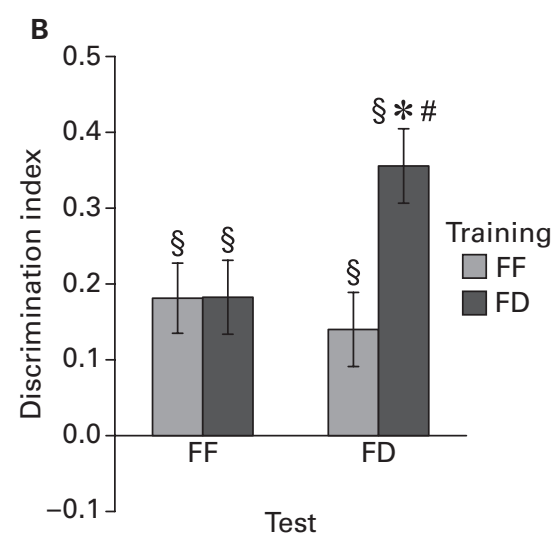

sampling is performed in an environment previously associated to a rewarding stimulus.

Control of the role of acute food deprivation. Two-way ANOVA did not reveal significant effects nor interaction between factors "feeding at training" and "feeding at test" in total exploration time. Figure 1B shows the D.I. measured $96 \mathrm{~h}$ after training as a function of feeding state (FF vs FD) before ORT sessions (training vs test). Two-way ANOVA revealed a significant effect of "feeding before training" $\left(F_{1,60}=5.06, p<0.05\right)$ and a significant interaction between "feeding before training" and "feeding before test" $\left(F_{1,60}=4.93, p<0.05\right)$. Simple effects analysis revealed that: $(1)$ D.I. of mice food-deprived before both training and test (FDFD) was higher than that of mice food-deprived before training but not before test (FD-FF; $t_{30}=-2.50, p<0.05$ ); (2) D.I. of mice food-deprived before both training and test (FD-FD) was higher than that of mice free-fed before training but not before test (FF-FD; $\left.t_{30}=-3.11, p<0.005\right)$; (3) D.I. of mice free-fed before both training and test (FF-FF) did not differ from that of mice free-fed before training but not before test (FF-FD); (4) D.I. of mice free-fed before both training and test (FF-FF) did not differ from that of mice free-fed before test but not before training (FD-FF). One sample $t$-tests revealed that D.I. was significantly above chance level for all groups (FD-FD: $t_{15}=7.25$, $p<0.0001$; FD-FF: $t_{15}=3.74, p<0.005$; FF-FD: $t_{15}=2.86$, $p<0.05$; FF-FF: $\left.t_{15}=3.92, p<0.005\right)$. These results indicate that acute food deprivation magnifies the enhancement of LTM duration promoted by sampling objects in the chocolate-associated context.

Control of the role of schedule of chocolate exposure. Oneway ANOVA for total exploration did not reveal significant group differences. Figure $\mathbf{1 C}$ shows the D.I. measured $96 \mathrm{~h}$ after training as a function of chocolate administration protocol. One-way ANOVA for D.I. revealed a significant group effect $\left(F_{2,45}=7.30, p<0.01\right)$. Tukey's post hoc analysis revealed that POST group had a lower D.I. respect to both PRE and PRE + POST groups which did not differ each other. One sample

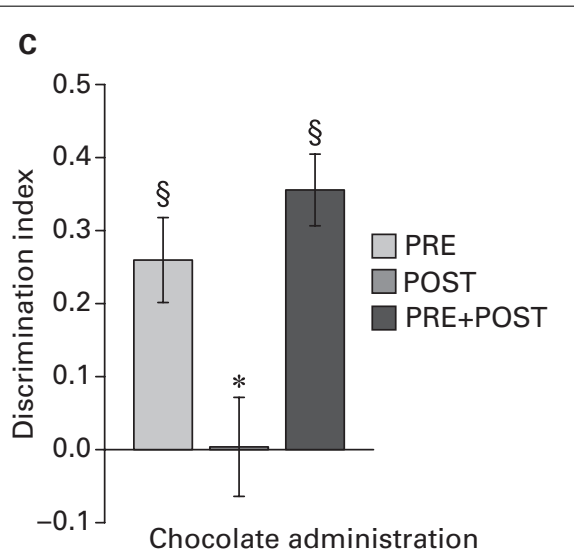

measured $96 \mathrm{~h}$ post-training ( ${ }^{*}$ vs FD-FF, $p<0.05$; ${ }^{*}$ vs FF-FD, $p<0.005 ;{ }^{\S}$ vs $0, p<0.0001$ ); (C) Discrimination index (mean \pm SEM) as a function of chocolate administration protocol measured $96 \mathrm{~h}$ post-training $\left(^{*} \mathrm{vs}\right.$ all,

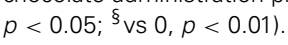

$t$-tests revealed that D.I. was significantly above chance level only for PRE $\left(t_{15}=4.46, p<0.001\right)$ and PRE $+\operatorname{POST}\left(t_{15}=7.30\right.$, $p<0.0001)$. These results indicate that the improvement of object memory observed in mice trained in the chocolate-associated context does not depend on the caloric and/or fat properties of chocolate.

\section{Experiment 2}

One-way ANOVA for total exploration did not reveal significant group differences. Figure 2A shows the D.I. measured $96 \mathrm{~h}$ after training in the object-paired environment as a function of the Rolipram dose injected immediately post-training. One-way ANOVA revealed a significant difference in D.I. between groups

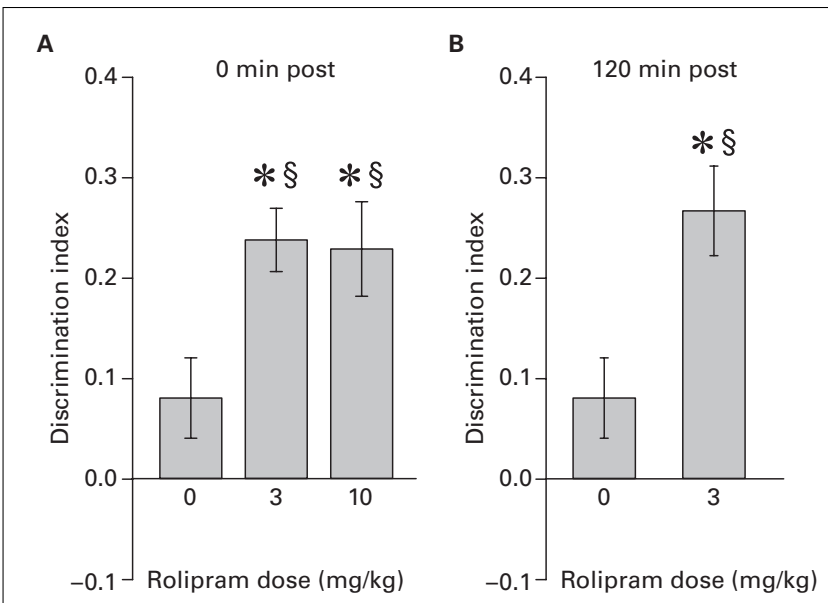

FIGURE 2 | (A) Discrimination index (mean \pm SEM) as a function of Rolipram dose $(0,3$, and $10 \mathrm{mg} / \mathrm{kg})$ injected immediately after training in the object-paired context measured $96 \mathrm{~h}$ post-training $\left(^{*} \mathrm{vs}\right.$ vehicle, $p<0.05$;

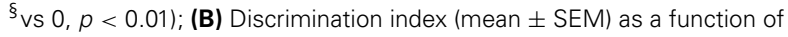
Rolipram dose (0 vs $3 \mathrm{mg} / \mathrm{kg}$ ) injected 120 min after training in the object-paired context measured $96 \mathrm{~h}$ post-training $\left({ }^{*} \mathrm{vs}\right.$ vehicle, $p<0.01$; $\S_{\text {Vs } 0, p<0.001)}$. 
$\left(F_{2,20}=4.51, p<0.05\right)$. Tukey's post hoc revealed that Rolipram enhanced D.I. respect to vehicle at both doses which did not differ one another. One sample $t$-tests revealed that D.I. was significantly above chance level only for Rolipram-treated groups [3 mg/kg $\left.\left(t_{7}=7.65, p<0.001\right) ; 10 \mathrm{mg} / \mathrm{kg}\left(t_{7}=4.82, p<0.01\right)\right]$. Figure 2B shows the D.I. measured $96 \mathrm{~h}$ after training in objectpaired environment in group injected $120 \mathrm{~min}$ post-training with $3 \mathrm{mg} / \mathrm{kg}$ of Rolipram and in the vehicle group. Two-sample $t$ test revealed that Rolipram-treated mice had higher D.I. respect to vehicle-treated mice $\left(t_{13}=-3.02, p<0.01\right)$. One sample $t$ test revealed that D.I. was significantly above chance level only for Rolipram-treated mice $\left(t_{7}=5.91, p<0.001\right)$. These results indicate that Rolipram enhances LTM duration either when injected immediately or $120 \mathrm{~min}$ post-training.

\section{INHIBITORY AVOIDANCE Experiment 3}

Figures 3A,B show step-through latency measured, respectively, $24 \mathrm{~h}$ and 1 week after training as a function of shock intensity. For $24 \mathrm{~h}$ test, Kruskal-Wallis ANOVA revealed a significant difference between groups $\left[H_{(2)}=23.25 ; p<0.001\right]$. Post hoc analysis revealed that groups trained with either shock intensities had a higher step-through latency respect to pseudo-trained group but did not differ one another $(0.35$ vs $0: W=26.5, p<0.001 ; 0.7$ vs $0: W=11, p<0.0001)$. For 1 week test, Kruskal-Wallis ANOVA revealed a significant difference between groups $\left[H_{(2)}=13.44\right.$; $p<0.01]$. Post hoc analysis revealed that the group trained with the 0.7 shock had a higher step-through latency respect to both pseudo- and 0.35 -trained groups ( 0.7 vs $0: W=41.5, p<0.001$; 0.7 vs $0.35: W=197.5, p<0.01)$ and that the latter groups did not differ one another. These results indicate that duration of fear LTM depends on the magnitude of negative arousal experienced during training.

\section{Experiment 4}

Figure 4A shows step-through latency measured 1 week after training with the 0.35 shock as a function of the Rolipram dose injected immediately post-training. Kruskal-Wallis ANOVA revealed a significant difference between groups $\left[H_{(2)}=7.55 ; p<0.05\right]$. Post hoc analysis revealed that only the group treated with $10 \mathrm{mg} / \mathrm{kg}$ of Rolipram had a step-through latency higher than vehicle-treated group $(W=23.5 ; p<0.01)$. Figure $4 B$ shows step-through latency measured 1 week after training with the 0.35 shock in group injected with vehicle or $10 \mathrm{mg} / \mathrm{kg}$ Rolipram $120 \mathrm{~min}$ post-training. Two-sample Wilcoxon test did not reveal significant difference between groups. Figure 4C shows step-through latency measured 1 week after training in groups injected immediately after pseudotraining with vehicle or $10 \mathrm{mg} / \mathrm{kg}$ Rolipram. Two-sample Wilcoxon test did not reveal significant difference between groups. Taken together, these results indicate that Rolipram enhances fear LTM only at the highest dose and only when injected immediately after training. Moreover, Rolipram has no effect on LTM duration in absence of shock.

\section{DISCUSSION}

Two are the main findings of the present study. The first is that long-term object memory is enhanced when sampling is performed under positive emotional arousal. Indeed, in the present experiments we demonstrated that mice remember a sampled object for $24 \mathrm{~h}$ but this memory can last $96 \mathrm{~h}$ if object sampling is performed in an environment previously associated with a rewarding stimulus. The second finding is that pharmacological stimulation of cAMP cascade enhances the duration of low emotional memories regardless of hedonic valence. Thus, we found that mice treated with Rolipram post-trial showed discrimination of an object sampled under low emotional arousal $96 \mathrm{~h}$ earlier and show IA of a context associated 1 week before with a low intensity shock.

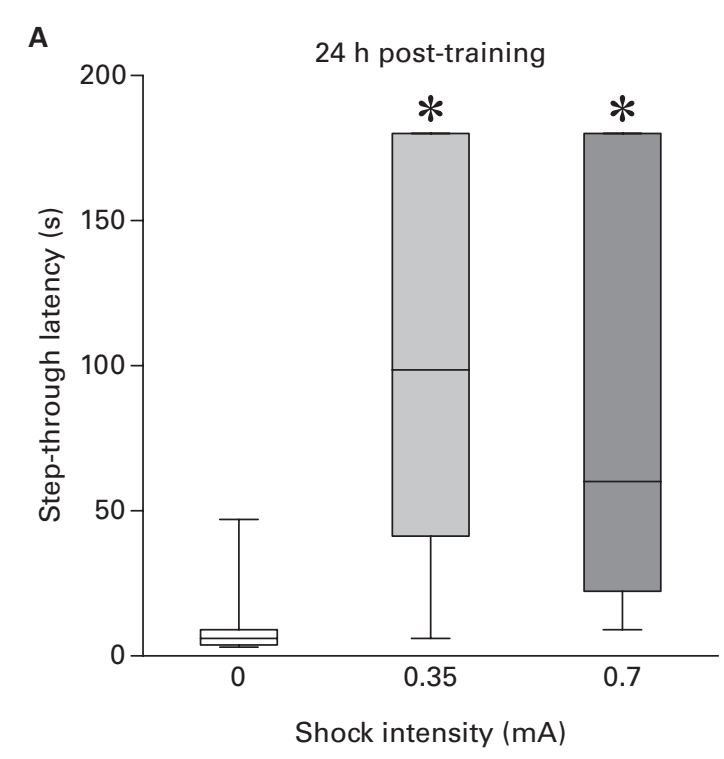

FIGURE 3 | (A) Step-through latency (median and inter-quartile range) as a function of foot-shock intensity ( $0.35 \mathrm{vs} 0.7 \mathrm{~mA})$ measured $24 \mathrm{~h}$ post-training ( ${ }^{*}$ vs $\left.0, p<0.001\right)$; (B) Step-through latency

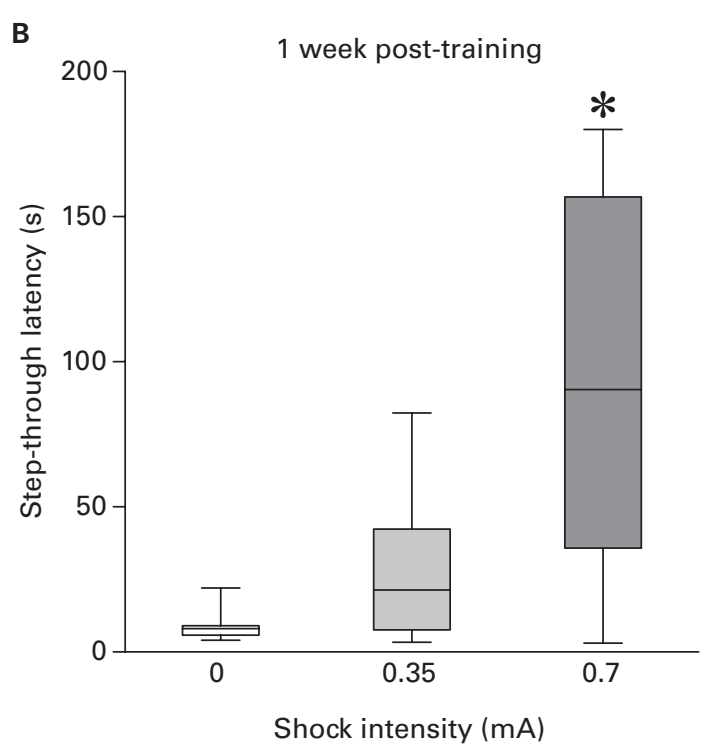

(median and inter-quartile range) as a function of foot-shock intensity (0.35 vs $0.7 \mathrm{~mA})$ measured 1 week post-training ( ${ }^{*} \mathrm{vs} 0$, $p<0.001)$. 


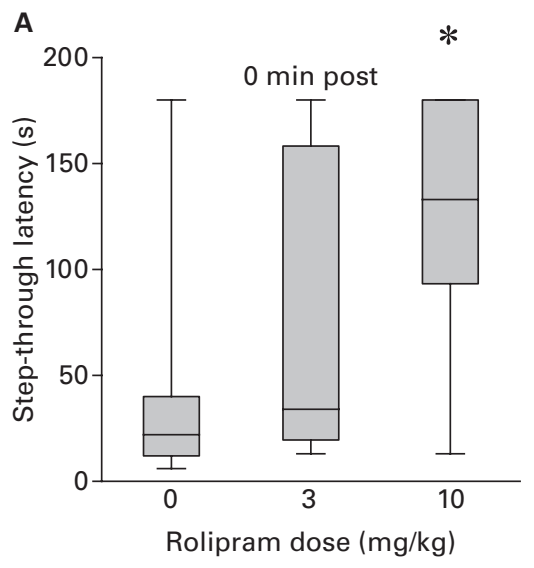

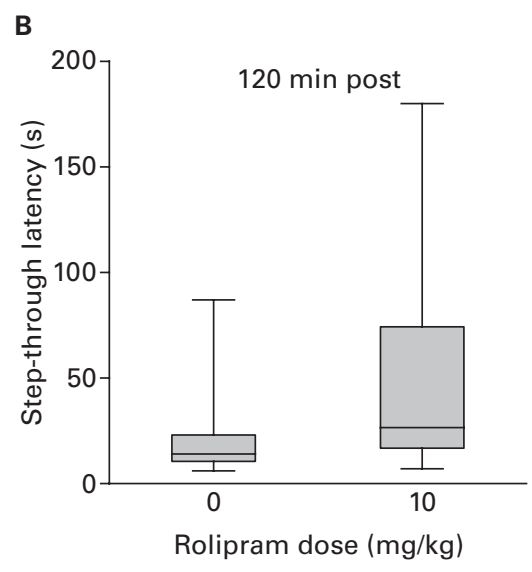

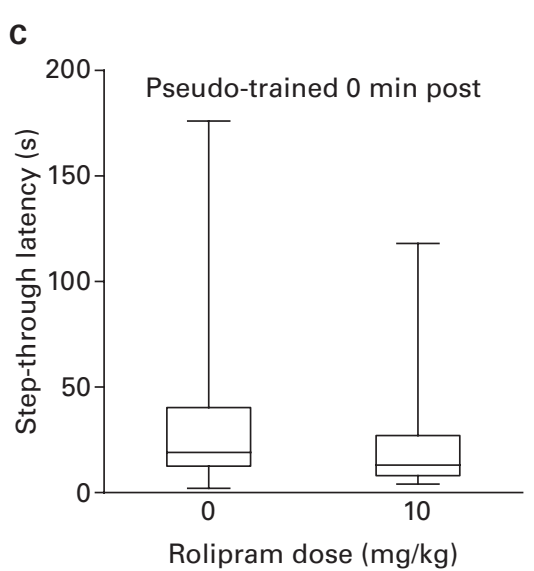

FIGURE 4 | (A) Step-through latency (median and inter-quartile range) as a function of foot-shock intensity ( 0.35 vs $0.7 \mathrm{~mA}$ ) measured 1 week posttraining (* vs all, $p<0.01$ ); (B) Step-through latency (median and inter-quartile range) as a function of Rolipram dose $(0,3$, and $10 \mathrm{mg} / \mathrm{kg})$ injected immediately after training with the $0.35 \mathrm{~mA}$ foot-shock measured 1 week post-training ( ${ }^{*}$ vs all, $p<0.05$ ); (C) Step-through latency (median and interquartile range) as a function of Rolipram dose (0 vs $10 \mathrm{mg} / \mathrm{kg}$ ) injected $120 \mathrm{~min}$ after training with the $0.35 \mathrm{~mA}$ foot-shock measured 1 week post-training.

\section{POSITIVE EMOTIONAL MODULATION OF LONG-TERM MEMORY}

The present study used a modified version of the widely used ORT that allowed modulation of the memory trace acquired during sampling by positive emotional arousal. To this aim we associated the experimental context with chocolate and then increased the motivational salience of the unconditioned stimulus by submitting the mice to a period of food deprivation immediately before ORT training and testing phases. According to several studies on reward anticipation, animals respond with a positive emotional arousal when they are returned into contexts that predicts the availability of highly palatable, caloric, and fat foods (Alcaro et al., 2007; Boissy et al., 2007; Alcaro and Panksepp, 2011). Mice trained and tested in a context associated with an inedible plastic stimulus were used as controls.

Both experimental and control mice discriminated the novel object when tested $24 \mathrm{~h}$ after training demonstrating intact memory of the previously sampled object. However, $96 \mathrm{~h}$ after training only mice trained and tested within the chocolate-associated context were still able to discriminate the novel object. These data support the view that experiences associated with a state of positive emotional arousal are consolidated in more lasting memories as reported for those associated with negative ones.

Control experiments reported by the present paper indicate that the LTM of the sampled object is modulated by the chocolateassociated context rather than by the chocolate feeding immediately after sampling. Indeed, mice that did receive chocolate on the object sampling session without previous chocolate-context pairing did not show discrimination of a novel object $96 \mathrm{~h}$ later, whereas mice that did not receive chocolate but were trained in a context previously associated with chocolate did. These findings rule out the influence of sugar or fat (Campolongo et al., 2009; Smith et al., 2011) on memory consolidation in our experimental conditions. Moreover, in our standard experimental condition chocolate was made available at the end of the sampling session to prevent negative emotional arousal associated with frustration (for review see Flaherty, 1996) as well as contingency between sample object and reward (Hughes, 2007).

Moreover, the data suggest that the chocolate-associated context was capable of promoting a lasting memory of the sampled object also in free-feeding mice; however, food-deprived mice showed a D.I. significantly higher than free-fed mice. This result supports the hypothesis that imposing $15 \mathrm{~h}$ of food deprivation before objects sampling increased the positive emotional arousal promoted by chocolate-associated context in line with the hypothesis that a physiological depletion enhances the incentive value of the unconditioned and conditioned goal stimuli (Berridge, 2004). Mice food-deprived before training but not before test showed lower discrimination than mice trained and tested in food deprivation strongly supporting a state-dependent learning. Finally, mice food-deprived only on test day (96 h) did not show any improvement of object discrimination in comparison with free-fed mice. The latter observation indicates a selective effect of the motivational state on memory formation and/or consolidation rather than on retrieval. Taken together, these findings support the view that acute food deprivation increased emotional arousal promoted by the context associated with chocolate, It is tempting to speculate that the condition of feeding deprivation invested the experience of re-entering into the reward-predicting context of a flashbulb-like quality (Brown and Kulik, 1977).

\section{NEGATIVE EMOTIONAL MODULATION OF LONG-TERM MEMORY}

Results from IA experiment confirm and extend to the mouse those of Bekinschtein et al. (2007, 2008a,b) in rats. Indeed, we found that training with both foot-shock intensities promoted LTM (i.e., IA at $24 \mathrm{~h}$ post-training) but only training with the stronger one induced a persistent LTM (1 week). The facilitating effect of negative arousal on LTM duration is in line with the above described effect of positive arousal, strongly suggesting that emotional arousal enhances LTM duration regardless of hedonic valence. 


\section{PHARMACOLOGICAL STIMULATION OF CAMP CASCADE ENHANCES PERSISTENCE OF LOW EMOTIONAL MEMORIES}

Pharmacological experiments evaluated the effects of single posttrial administration of Rolipram on retention of low emotional memories. We used two different tests: ORT and step-through IA. For both tests we considered protocols capable of promoting LTM traces of moderate persistence. In the case of ORT, we used mice trained and tested in the context associated with the plastic stimulus because these animals show retention $24 \mathrm{~h}$ but not $96 \mathrm{~h}$ after training. In the case of IA, we performed a preliminary experiment using 0.35 and $0.70 \mathrm{~mA}$ and chose the lower shock intensity that was effective in increasing step-through latencies $24 \mathrm{~h}$ but not 1 week post-trial.

The effects of Rolipram were tested at the time-points at which no retention was observable ( $96 \mathrm{~h}$ for ORT and 1 week for IA) in vehicle-treated mice. In both cases we found retention in Rolipram-treated mice. Rolipram is a phosphodiesterase type IV inhibitor that, at the doses used in the present experiments, promotes a rapid rise of cAMP concentration in the mouse brain (Randt et al., 1982). The treatment, in both cases, was performed immediately after a single training session, when the memory traces are under consolidation and susceptible of manipulation (McGaugh, 1966). Therefore, the present results suggest that an increase of brain cAMP concentrations during consolidation mimics the effects of high emotional arousal on persistence of long-term memories.

\section{REFERENCES}

Alcaro, A., Huber, R., and Panksepp, J. (2007). Behavioral functions of the mesolimbic dopaminergic system: an affective neuroethological perspective. Brain Res. Rev. 56, 283-321.

Alcaro, A., and Panksepp, J. (2011). The SEEKING mind: primal neuroaffective substrates for appetitive incentive states and their pathological dynamics in addictions and depression. Neurosci. Biobehav. Rev. 35, 1805-1820.

Baarendse, P. J., van Grootheest, G., Jansen, R. F., Pieneman, A. W., Ogren, S. O., Verhage, M., and Stiedl, O. (2008). Differential involvement of the dorsal hippocampus in passive avoidance in $\mathrm{C} 57 \mathrm{bl} / 6 \mathrm{~J}$ and $\mathrm{DBA} / 2 \mathrm{~J}$. Hippocampus 18, 11-19.

Bekinschtein, P., Cammarota, M., Igaz, L. M., Bevilaqua, L. R., Izquierdo, I., and Medina, J. H. (2007). Persistence of long-term memory storage requires a late protein synthesisand BDNF-dependent phase in the hippocampus. Neuron 53, 261-277.

Bekinschtein, P., Cammarota, M., Izquierdo, I., and Medina, J. H. (2008a). BDNF and memory formation and storage. Neuroscientist 14, 147-156.

Bekinschtein, P., Cammarota, M., Katche, C., Slipczuk, L., Rossato, J. I., Goldin, A., Izquierdo, I., and Medina,
J. H. (2008b). BDNF is essential to promote persistence of long-term memory storage. Proc. Natl. Acad.

Berridge, K. C. (2004). Motivation concepts in behavioral neuroscience. Physiol. Behav. 81, 179-209.

Boccia, M. M., Acosta, G. B., Blake, M. G., and Baratti, C. M. (2004). Memory consolidation and reconsolidation of an inhibitory avoidance response in mice: effects of i.c.v. injections of hemicholinium-3. Neuroscience $124,735-741$.

Boissy, A., Manteuffel, G., Jensen, M. B., Moe, R. O., Spruijt, B., Keeling, L. J., Winckler, C., Forkman, B., Dimitrov, I., Langbein, J., Bakken, M., Veissier, I., and Aubert, A. (2007). Assessment of positive emotions in animals to improve their welfare. Physiol. Behav. 92, 375-397.

Brown, B., and Kulik, J. (1977). Flashbulb memories. Cognition 5, 73-99.

Campolongo, P., Roozendaal, B., Trezza, V., Cuomo, V., Astarita, G., Fu, J., McGaugh, J. L., and Piomelli, D. (2009). Fat-induced satiety factor oleoylethanolamide enhances memory consolidation. Proc. Natl. Acad. Sci. U.S.A. 106, 8027-8031.

Dudai, Y. (1996). Consolidation: fragility on the road to the engram. Neuron 17, 367-370.

Dudai, Y. (2002). Molecular bases of long-term memories: a question of Sci. U.S.A. 105, 2711-2716.

Nonetheless, major differences were found in the effects of Rolipram on ORT and IA retention. Thus, only the high dose of the phosphodiesterase inhibitor was effecting in enhancing stepthrough latencies 1 week after IA training, whereas the lower dose was already capable to enhance D.I. to the level of the high dose in mice tested $96 \mathrm{~h}$ after ORT training. The most economic explanation of this difference could be that step-through latencies and D.I. were tested at different time points after training. Indeed, if the pharmacological manipulation is increasing duration of the memory traces, a lower dose could be sufficient to produce a $96-\mathrm{h}$ duration whereas only larger doses would induce 1 week-lasting memories. Another difference between the effects of Rolipram on retention in the two conditions was that the drug improved object memory when administered 120 min post-trial, whereas it improved IA retention only when administered immediately posttrial. It should be noted that Rolipram improves object memory in rats if injected $3 \mathrm{~h}$, but not immediately, after sampling (Rutten et al., 2007). However, the dose administered in the present study is much more higher than the one used by Rutten et al. (2007). Thus, the observed efficacy of immediate post-sampling administration could depend on the half-life of Rolipram extending up to $120 \mathrm{~min}$. On the other hand, such hypothesis fails to explain why an even higher dose $(10 \mathrm{mg} / \mathrm{kg})$ enhances IA retention if administered immediately, but not $120 \mathrm{~min}$, after training. This may suggest that newly formed non-aversive memories remain susceptible to cAMP stimulation for a longer time respect to aversive ones.

persistence. Curr. Opin. Neurobiol. $12,211-216$.

Dudai, Y. (2004). The neurobiology of consolidations, or, how stable is the engram? Annu. Rev. Psychol. 55, 51-86.

Dudai, Y., and Eisenberg, M. (2004). Rites of passage of the engram: reconsolidation and the lingering consolidation hypothesis. Neuron 44, 93-100.

Ennaceur, A. (2010). One-trial object recognition in rats and mice: methodological and theoretical issues. Behav. Brain Res. 215, 244-254.

Ennaceur, A., and Delacour, J. (1988). A new one-trial test for neurobiological studies of memory in rats. 1: behavioral data. Behav. Brain Res. 31, 47-59.

Flaherty, C. F. (1996). Incentive Relativity. Cambridge: Cambridge University Press.

Fox, J. (2005). The R commander: a basic-statistics graphical user interface to R. J. Stat. Softw. 14, 1-42.

Hughes, R. N. (2007). Neotic preferences in laboratory rodents: issues, assessment and substrates. Neurosci. Biobehav. Rev. 31, 441-464.

Lechner, H. A., Squire, L. R., and Byrne, J. H. (1999). 100 years of consolidation-remembering Müller and Pilzecker. Learn. Mem. 6 , 77-87.
McGaugh, J. L. (1966). Time-dependent processes in memory storage. Science 153, 1351-1358.

McGaugh, J. L. (2006). Make mild moments memorable: add a little arousal. Trends Cogn. Sci. 10, 345-347.

Nilsson, M., Hansson, S., Carlsson, A., and Carlsson, M. L. (2007). Differential effects of the $N$-methyl-Daspartate receptor antagonist MK801 on different stages of object recognition memory in mice. Neuroscience 149, 123-130.

Randt, C. T., Judge, M. E., Bonnet, K. A., and Quartermain, D. (1982). Brain cyclic AMP and memory in mice. Pharmacol. Biochem. Behav. 17, 677-680.

Rossato, J. I., Bevilaqua, L. R., Izquierdo, I., Medina, J. H., and Cammarota, M. (2009). Dopamine controls persistence of long-term memory storage. Science 325, 1017-1020.

Rutten, K., Prickaerts, J., and Blokland, A. (2006). Rolipram reverses scopolamine-induced and timedependent memory deficits in object recognition by different mechanisms of action. Neurobiol. Learn. Mem. 85, 132-138.

Rutten, K., Prickaerts, J., Hendrix, M., van der Staay, F. J., Sik, A. and Blokland, A. (2007). Timedependent involvement of cAMP and cGMP in consolidation of object 
memory: studies using selective phosphodiesterase type 2, 4 and 5 inhibitors. Eur. J. Pharmacol. 558, 107-112.

Sik, A., van Nieuwehuyzen, P., Prickaerts, J., and Blokland, A. (2003). Performance of different mouse strains in an object recognition task. Behav. Brain Res. 147, 49-54.

Smith, M. A., Riby, L. M., Eekelen, J. A., and Foster, J. K. (2011). Glucose enhancement of human memory: a comprehensive research review of the glucose memory facilitation effect. Neurosci. Biobehav. Rev. 35, 770-783.

Conflict of Interest Statement: The authors declare that the research was conducted in the absence of any commercial or financial relationships that could be construed as a potential conflict of interest.

Received: 21 September 2011; paper pending published: 10 October 2011; accepted: 10 December 2011; published online: 23 December 2011.

Citation: Cruciani F, Berardi A Cabib S and Conversi D (2011) Positive and negative emotional arousal increases duration of memory traces: common and independent mechanisms.
Front. Behav. Neurosci. 5:86. doi: 10.3389/fnbeh.2011.00086

Copyright (c) 2011 Cruciani, Berardi, Cabib and Conversi. This is an openaccess article distributed under the terms of the Creative Commons Attribution Non Commercial License, which permits non-commercial use, distribution, and reproduction in other forums, provided the original authors and source are credited. 